\title{
The significance of nanomedicine in brain-targeted drug delivery: crossing blood-brain barriers
}

\begin{abstract}
At present, the brain ailments are among the most complex disease to treat since therapies are strictly hindered through the highly selective blood-brain barrier (BBB). The efficacy of CNS acting therapeutics is determined by their ability to cross the BBB at therapeutic dose. Therefore, there is an urgent need for strategies that enable CNS therapeutic to cross BBB effectively hitherto frozen mainly of BBB. The nanomedicine represents a cutting edge tool in the field of biomedicine and promising addition to the spectrum of brain-targeted drug delivery. The promises revolve around the biocompatibility and unique versatility of site-specific drug delivery. A broad variety of CNS acting therapeutics can be entrapped in polymeric matrix, which further can be surface engineered with brain-specific ligands to facilitate the delivery of therapeutics across BBB. The scope of this communication presents recent advancements in the field of nanomedicine in context to brain-targeted drug delivery, with an eye toward the opportunity for translation from bench to bedside.
\end{abstract}

Volume 5 Issue 5 - 2017

\author{
Dhirender Singh,' Bhavesh Kevadiya, ${ }^{2}$ \\ Kalpana Nagpal, ${ }^{3}$ Navneet Sharma, ${ }^{4}$ \\ Sandipkumar Patel' \\ 'Navinta LLC, USA \\ ${ }^{2}$ Department of Pharmacology and Experimental Neuroscience, \\ University of Nebraska, USA \\ ${ }^{3}$ Amity Institute of Pharmacy, Amity University, India \\ ${ }^{4}$ Patheon Inc, USA
}

Correspondence: Dhirender Singh, Navinta LLC, Ewing, USA, Tel 402-359-37I3 Email chauhandhiru@gmail.com

Received: May 24, 2017 | Published: July 03, 2017

\section{Introduction}

Last two decades have seen a tremendous and fascinating advancement in the field of drug development. Despite the progression in the scientific technology, the diseases of central nervous system (CNS) present a formidable challenge to the clinicians. Prospects to improve quality of life and halt or ameliorate age-related neurodegenerative diseases like Dementia, Alzheimer's, Parkinson's etc. are still far to measure. At present, around 1.5 billion people worldwide are suffering from one or another CNS disease . ${ }^{1}$ As reviewed in 2017, around 47.5 million people around the globe are living with dementia. The prevalence of dementia is anticipated to be 75.63 million in 2030 , reaching to 135.46 million by 2050 , which is higher than original number estimated in the 2009 World Alzheimer report .2 Specifically, in US about 5.2 million people are suffering from Alzheimer's disease (AD), which has been cited as the sixth leading cause of death, and ranked fifth among those aged 65 years and older. Unless medical breakthroughs are made to favor the pharmacokinetics and pharmacodynamics of experimental therapeutics, it is estimated that by 2050 , the number of AD patients may nearly triple from 5 million to a projected 14 million ${ }^{3,4}$ Of the all CNS related diseases, brain tumor is among the most challenging and lethal. Updated in January 2017, nearly 700,000 people in U.S. are living with CNS related tumor. It is estimated that nearly 80,000 cases of primary brain tumor would be diagnosed by the end of 2017 . One third of the $32 \%$ of the diagnosed cases are anticipated to be malignant, and nearly 53,000 will be non-malignant. Sadly, by the end of 2017, nearly 17,000 people with CNS brain tumor will lose their battle with life. ${ }^{5}$

Regardless of the scientific researches and technologies, no effective therapies are out in clinic for most of the brain tumors. The failure of vast majority of novel and current experiential therapeutics to reach/target the brain at a reasonable effective dose remains a major challenge. Despite great stride in understanding of the brain biology, from cellular to behavioral levels, the advances in basic science have not yet been fully developed in an interdisciplinary way, and a definitive translation from bench to bedside is still uncertain . ${ }^{6}$ The scope of current communication focuses current challenges in braindrug delivery, and significance of nanomedicine in crossing BBB, their downside and future prospects are also discussed.

\section{The challenges of BBB in brain drug delivery}

It is now well established that vasculature within brain capillaries are made of a unique barricade, coined blood brain barrier (BBB) which segregates the brain from harmful substances while impeding the systemic delivery of pharmaceuticals from the blood into the brain . ${ }^{7}$ Undisputedly, $98 \%$ of CNS acting small molecules and $100 \%$ of large-molecules drugs i.e. proteins, peptide, siRNA, monoclonal antibodies etc. are unable to prevail brain at a therapeutic effective concentration, owing to BBB hindrance. Consequently, the fences of BBB limit the progression in an effective pharmacological therapy in number of neurological disorders. It should be noted that not only mechanical barricade of BBB, but the presence of efflux pump such as P-glycoprotein (P-gp) also restricts the entry of the therapeutics to the brain parenchyma, while allowing entry of molecules such as essential nutrients for brain homeostasis. Despite possessing the required physicochemical properties to effectively permeate and get access to the brain parenchyma at first instance, pharmaceuticals which are recognized by P-gp efflux pump may get expelled out.$^{8,9}$

However, the BBB is not only a mechanical barricade to the pharmaceuticals, but also a dynamic biological membrane, in which carrier and/or receptor-mediated transport and active metabolism takes place. Nevertheless, large molecules such as cytokines and neurotrophins enter the brain via receptor-mediated endocytosis, whereas specific transporters mediate the entrance of essential nutrients, including amino acids, glucose and ketone bodies.$^{10}$ In general, under normal physiological conditions, few substances are capable to surpass the BBB by carrier-mediated transport, receptormediated endocytosis, and scantly through passive diffusion and adsorptive transcytosis. However, the possibility for molecule to diffuse passively across BBB is very limited, and confined to lipid soluble molecules with a molecular weight of $<400 \mathrm{Da} . .^{10}$

However, torealize adsorptive-endocytosis, itrequires an interaction between the brain capillaries cell surface and ligands, such as protein or macromolecules in the bloodstream. Generally, interactions are triggered by electrostatic interaction between negatively charged cell surface and positively charged ligands. Following the interactions, in most cases, clathrin-dependent endocytosis mediates the adsorptive transcytosis. This mode of transport, however, is unidirectional, from 
blood to brain, and specific to cationic ligands. Moreover, the extent to which adsorptive transcytosis mediate passage substances across the BBB is unclear, and still under investigation. Undisputedly, the majority of uptake to brain parenchyma surpassing BBB is mediated by carrier-mediated and receptor-mediated transport, where the molecule possesses high affinity to specific carrier or receptor expressed at the brain capillaries endothelial cells.$^{11,12}$

For example, water soluble substrates such as hexoses, glucose, are bound and transport through the specific receptor i.e. glucose transporter type 1 (GLUT1), whereas phenylalanine and over ten large neutral amino acids are transported to the brain through large neutral amino-acid transporter type 1 (LAT1).${ }^{13}$ Several of the natural mechanisms involved in BBB crossing of endogenous molecules can be employed for the development of brain-targeted drug delivery. Transport of L-DOPA across the BBB via the LAT1 carrier is a classic example where drug structure can be modified to mimic the endogenous substrate of transporter. L-DOPA is a precursor of dopamine used in Parkinson's disease.${ }^{14}$ However, after successfully accessing to the brain parenchyma, most therapeutic molecules are taken up by brain capillary endothelial cells, mostly via clathrinmediated endocytosis.

The uptake via such mechanism eventually leads to delivery of the drug into acid filled endo-lysosomal compartments. The low $\mathrm{pH}$ and presence of degrading enzymes in such compartments eventually degrade the drugs; possessing a major threat to the active drug moiety, particularly biotherapeutics.$^{15}$ Thus, given such premises, there is an urgent need to develop new strategies to surpass BBB fence effectively. The drug molecule needs to be protected from degradation, rapid/ extensive metabolism, and clearance within systemic circulation; delivered across BBB with high efficiency; and accumulate sufficiently at the site of action. To address the challenge, the focus of brain researches has been directed towards the development of effective strategies to surpass the BBB over the past three decades. To this end, myriads of strategies to get in the brain have been employed, such as chemical drug delivery.${ }^{16}$ osmotic disruption of BBB.${ }^{16}$ ultrasound.${ }^{17}$ and nanomedicine based approach.$^{18}$ among others.

\section{Nanomedicine:Trojan- Horse to brain drug delivery}

Nanomedicine, which is a formulation of polymeric/lipidic precursor/carrier, can entrap a wide a variety of therapeutics. Generally, size of nanomedicine is in the range of 50-200 nm, which however, is too large to cross BBB through free diffusion.$^{19}$ To address the issue, outer surface of NPs is further engineered with various targeting ligands that mimic endogenous substances, and thus, gain access to brain parenchyma using receptor-mediated endocytosis .$^{20}$ It is predicted that nanomedicine can deliver entrapped drug in to the brain parenchyma at least in two ways;

a. By accumulating entrapped drug at the luminal surface of the cells of BBB that leads to a local high concentration gradient between blood and brain, which is higher than achieved through intravenous administration of the free drugs. The accumulation of concentration gradient further facilitates the passive diffusion of the free drug across BBB, provided the drug molecule possesses the required permeability characteristics. This strategy, however, can be followed or not by their subsequent successful passage through targeted cells;

b. Contrary, intact nanomedicine with entrapped cargo can move them into brain parenchyma. The later feature can be realized by enabling nanomedicine to target the endothelial cells of brain capillaries and their subsequent transcellular passage across the $\mathrm{BBB} \cdot{ }^{21}$
In this context, a vast majority of brain-targeted ligands were identified, expressed in various in vitro models, and screened for their efficiency to deliver drug in brain parenchyma, hitherto frozen as of poor BBB penetrance. As an example of such a system, a cyclic A7R peptide (cA7R) was identified and synthesized that could specifically bind to vascular endothelial growth factor receptor 2 (VEGFR2) and neuropilin-1 (NRP-1). The conjugation of cA7R peptide on to the surface of doxorubicin loaded liposomes resulted in higher accumulation, and antiglioma effect in glioma bearing mice model .${ }^{22}$ In a recent study, solid-lipid nanoparticles decorated with human apolipoprotein E (ApoE) were evaluated for their adaptability to surpass BBB. The administration of nanomedicine via pulmonary route demonstrated improved bioavailability, compared with unmodified counterpart ${ }^{20}$

Similarly, a synthetic seven-peptide (sequenced HAIYPRH, T7) that was screened by a phage displayed a higher affinity for transferrin receptors (TfR); a carrier protein known to express abundantly over the brain micro vascular endothelial cells. Conjugation of peptide with lipid nanoparticles that were loaded with siRNA, exhibited a significant higher accumulation, and hence higher therapeutic efficacy compared with unmodified nanoparticles.$^{23}$ Interestingly, in a very novel approach, mini cells were derived from chromosomal deletion mutant strain of Salmonella enterica serovar Typhimurium (S. Typhimurium) bacteria, purified, and packed with anticancer drug doxorubicin. The safety and efficacy of the epidermal growth factor receptor (EGFR) targeted mini-cells were evaluated in spontaneous brain tumors in dogs; a comparative model of human brain cancers. On the premises of pharmacological activity observed in preclinical species, a strong potential for clinical implication was prospected. Subsequently, a Phase 1 clinical study of EGFR targeted mini-cells packed with doxorubicin is under investigation for the treatment of glioblastoma.$^{24,25}$

Despite strong potential exhibited by brain directed nanomedicine, their accumulation at brain site, before they are cleared via reticuloendothelial system (RES), is a major obstruction in their way to clinic.${ }^{26}$ However, to address this issue, magnetic-targeting has emerged as cutting edge tool in the field of brain drug delivery. Under the influence of external magnetic force, the magnetic nanoparticles (MNPs); the polymeric precursor of 2 to $10 \mathrm{~nm}$ iron oxide super paramagnetic nanoparticles (magnetite or maghemite) in conjunction with therapeutic agent can be dragged to the site of action, brain in particular. Following brain region accumulation, the MNPs either create a local high concentration gradient of entrapped cargo at the luminal side of BBB that may facilitate passive diffusion of free drugs, or MNPs can move themselves across BBB. ${ }^{27,28}$ However, later task can be realized enabling MNPs targeting to endothelial cells of brain capillaries. To support the notion of passive accumulation, magnetictargeting was successfully employed to deliver rhodamine 123 into brain parenchyma. ${ }^{29}$ Being a P-gp substrate and hydrophilic in nature, the brain delivery of dye is otherwise restricted by BBB.$^{29}$ To realize the later task of dual-targeting (i.e. local accumulation followed by transcellular passage), magnetic PLGA MNPs modified with transferrin receptor binding peptide $\mathrm{T} 7$, under the guidance of external magnetic Field, more than 5-folds increase in brain accumulation of entrapped drugs (i.e. curcumin and paclitaxel) compared to nontargeting MNPs.$^{30}$

\section{Conclusion}

Despite growing evidence that nanomedicine hold great potential, especially in the field of site-specific delivery, translation of these studies to clinical application still presents a tremendous challenge 
to the scientists. Nanomedicine based strategies invariably involves the combination of CNS acting agents and brain targeting system; both of which have the potential for systemic and neurotoxicity. The disruptions of BBB, along with many pitfalls need to be resolved. Interdisciplinary investigations bringing together the fields of chemistry, biology, and engineering can yield effective way to circumvent BBB for improving global brain health.

\section{Conflicts of interest}

Author declares there is no Conflict of Interest.

\section{Acknowledgments}

None.

\section{References}

1. Kanwar JR, Sriramoju B, Kanwar RK Neurological disorders and therapeutics targeted to surmount the blood-brain barrier. Int $J$ Nanomedicine. 2012;7:3259-3278.

2. The Epidemiology and Impact of Dementia. WHO, Switzerland.

3. Policy Brief for Heads of Government. The Global Impact of Dementia 2013-2050 Alzheimer's Disease International (ADI).

4. Fonseca-Santos B, Gremião MP, Chorilli M Nanotechnology-based drug delivery systems for the treatment of Alzheimer's disease. Int $J$ Nanomedicine. 2015;10:4981-5003.

5. RL Siegel, KD Miller, A Jemal, Cancer statistics. CA: A Cancer Journal for Clinicians. 2017;67(1):7-30.

6. Meng J, Agrahari V, Youm I Advances in Targeted Drug Delivery Approaches for the Central Nervous System Tumors: The Inspiration of Nanobiotechnology. J Neuroimmune Pharmacol. 2017;12(1):84-98.

7. Zhang TT, Li W, Meng G, Wang P, Liao W Strategies for transporting nanoparticles across the blood-brain barrier. Biomater Sci. 2016;4(2):219-229.

8. Joshi CR, Labhasetwar V, Ghorpade A Destination Brain: the Past, Present, and Future of Therapeutic Gene Delivery. J Neuroimmune Pharmacol. 2017;12(1):51-83.

9. Pardridge WM The Blood-Brain Barrier: Bottleneck in Brain Drug Development. NeuroRx. 2005;2(1):3-14.

10. Barar J, Rafi MA, Pourseif MM et al. Blood-brain barrier transport machineries and targeted therapy of brain diseases. Bioimpacts. 2016;6(4):225-248.

11. Schenk GJ1, de Vries HE Altered blood-brain barrier transport in neuroinflammatory disorders. Drug Discov Today Technol. 2016;20:5-11.

12. Brzica H, Abdullahi W, Ibbotson $\mathrm{K}$ et al. Role of Transporters in Central Nervous System Drug Delivery and Blood-Brain Barrier Protection: Relevance to Treatment of Stroke. J Cent Nerv Syst Dis. 2017;9:11795735-17693802.

13. Hayashi K, Jutabha P, Maeda S, Supak Y, Ouchi M, et al. LAT1 acts as a crucial transporter of amino acids in human thymic carcinoma cells. $J$ Pharmacol Sci. 2016;132(3):201-204.
14. Mikitsh JL, Chacko AM Pathways for Small Molecule Delivery to the Central Nervous System Across the Blood-Brain Barrier. Perspect Medicin Chem. 2014;6:11-24.

15. Wu F, Yao PJ Clathrin-mediated endocytosis and Alzheimer's disease: an update. Ageing Res Rev. 2009;8(3):147-149.

16. Lv W, Zhang G, Barinka $\mathrm{C}$ et al. Design and Synthesis of Mercaptoacetamides as Potent, Selective, and Brain Permeable Histone Deacetylase 6 Inhibitors. ACS Med Chem Lett. 2017;8(5):510-515.

17. Mead BP, Kim N, Miller GW et al. Novel Focused Ultrasound Gene Therapy Approach Noninvasively Restores Dopaminergic Neuron Function in a Rat Parkinson's Disease Model. Nano Lett. 2017;17(6):3533-3542.

18. Song E, Gaudin A, King AR et al. Surface chemistry governs cellular tropism of nanoparticles in the brain. Nat Commun. 2017;8:15322.

19. Olivier JC Drug Transport to Brain with Targeted Nanoparticles. NeuroRx. 2005;2(1):108-119.

20. Dal Magro R, Ornaghi F, Cambianica I et al. ApoE-modified solid lipid nanoparticles: A feasible strategy to cross the blood-brain barrier. $J$ Control Release. 2017;249:103-110.

21. L Martin-Banderas, MA Holgado, JL Venero et al. Nanostructures for drug delivery to the brain. Curr Med Chem. 2011;18(34):5303-5321.

22. M Ying, Q Shen, C Zhan et al. A stabilized peptide ligand for multifunctional glioma targeted drug delivery. $J$ Contr Rel. 2016;243:86-98.

23. Wei L, Guo XY, T Yang et al. Brain tumor-targeted therapy by systemic delivery of siRNA with Transferrin receptor-mediated core-shell nanoparticles. Inter J Pharm. 2016;510(1):394-405.

24. JA Mac Diarmid, NB Mugridge, JC Weiss et al. Bacterially derived $400 \mathrm{~nm}$ particles for encapsulation and cancer cell targeting of chemotherapeutics. Cancer Cell. 2007;11(5):431-445.

25. JA MacDiarmid, V Langova, D Bailey et al. Targeted Doxorubicin Delivery to Brain Tumors via Minicells: Proof of Principle Using Dogs with Spontaneously Occurring Tumors as a Model. PLOS ONE. 2016;11(4): e0151832.

26. C Saraiva, C Praça, R. Ferreira et al. Nanoparticle-mediated brain drug delivery: Overcoming blood-brain barrier to treat neurodegenerative diseases. J Control Release. 2016;235:34-47.

27. D Singh, JM McMillan, XM Liu et al. Formulation design facilitates magnetic nanoparticles delivery to diseased cells and tissues. Future medicine. 2014;9(3):469-485.

28. D Singh, JM McMillan, AV Kabanov et al. Bench-to-bedside translation of magnetic nanoparticles. Nanomedicine (London). 2014;9(4):501-516.

29. B Kirthivasan, D Singh, MM Bommana et al. Active brain targeting of a fluorescent $\mathrm{P}-\mathrm{gp}$ substrate using polymeric magnetic nanocarrier system. Nanotechnology. 2012;23(25):255102.

30. Y Cui, M Zhang, F Zeng et al. Dual-Targeting Magnetic PLGA Nanoparticles for Co delivery of Paclitaxel and Curcumin for Brain Tumor Therapy. ACS Applied Materials \& Interfaces. 2016;8(47):32159-32169. 